International Mathematical Forum, 4, 2009, no. 44, 2199 - 2204

\title{
The Characterization of Congruences on Additive Inverse Semirings ${ }^{1}$
}

\author{
Wenting Xiong, Yuanlan Zhou and Xiaofeng Zhu \\ Department of Mathematics, Jiangxi Normal University \\ Jiangxi, Nanchang, 330022, P. R. China \\ ylzhou185@163.com
}

\begin{abstract}
In this paper, the congruence pairs on additive inverse semirings are defined. Some results about congruences and congruence pairs on additive inverse semirings are given. Our main conclusion is that congruences on semirings are ascertained by congruence pairs on it uniquely.
\end{abstract}

Keywords: additive inverse semiring, congruence, congruence pair

\section{Introduction}

A semiring $S$ is defined as an algebra system $(S,+, \cdot)$, such that $(S,+)$ and $(S, \cdot)$ are semigroups connected by $a(b+c)=a b+a c$ and $(b+c) a=b a+c a$ for all $a, b, c \in S$. A semiring $S$ is a skew ring if its additive reduct $(S,+)$ is a group. A semiring $S$ is an additive inverse semiring if $(S,+)$ is an inverse semigroup.

A nonempty subset $A$ of a semiring $S$ is called an ideal of $S$ if $a+b \in$ $A$, sa, as $\in A$ for all $a, b \in A, s \in S$. If $S$ is an inverse semiring, we denote $-a$ the unique additive inverse of $a, E^{+}(S)$ the set of all additive idempotents. Easily, we can prove if $S$ is an additive inverse semiring, then $E^{+}(S)$ is an ideal of $S$.

A relation $\mathrm{R}$ on the semiring $S$ is called compatible (with the operation on $S)$ if

$$
(\forall a, b, c, d \in S) \quad(a, b),(c, d) \in R \Rightarrow(a+c, b+d),(a c, b d) \in R .
$$

A relation $\rho$ on $S$ is called a congruence if and only if it is an equivalence relation and compatible with the operation on $S$.

\footnotetext{
${ }^{1}$ The research is supported by the NSF of Jiangxi Province(0611051); the SF of Education Department of Jiangxi Province(GJJ09459) and the SF of Jiangxi Normal University(1905).
} 
Semirings with special additive reduct are studied in ([5], [9], [6], [4], [3], [7]). The congruences and the congruence pairs on an inverse semigroup $([2])$ and orthorings ([8]) are characterized respectively. Other definitions and results about semigroups and semirings are referred to [2] and [1]. In this paper, we only considered the additive inverse semirings and we will generalize some results about congruences and congruence pairs on additive inverse semirings and give some characterizations and structure of additive inverse semirings.

\section{Characterizations and Structure}

Let $\rho$ be a congruence on an additive inverse semiring $S$. The restriction of $\rho$ to $E^{+}(S)$ is a congruence on $E^{+}(S)$, which we call the trace of $\rho$ and write as $\tau=\operatorname{tr} \rho$.

Definition 2.1. Let $S$ be an additive inverse semiring, $\sigma$ a congruence on $E^{+}(S) . \sigma$ is normal, in the sense that

(i) $e \sigma f \Rightarrow(\forall a \in S) \quad(-a+e+a) \sigma(-a+f+a)$;

(ii) $e \sigma f \Rightarrow(\forall c \in S) \quad e c \sigma f c$ and $c e \sigma c f$.

Clearly, $\operatorname{tr} \rho$ is a normal congruence on $E^{+}(S)$ Since we certainly have $-a+e+a,-a+f+a, e c, f c, c e, c f$ are in $E^{+}(S)$.

Easily we can obtain the following result:

Proposition 2.2. Let $\rho$ be a congruence on an additive inverse semiring $S$. Then $S / \rho$ is a skew-ring if and only if $\operatorname{tr} \rho=E^{+}(S) \times E^{+}(S)$.

Next, we define $N=\operatorname{ker} \rho$, the kernel of $\rho$, to be the union of all the additive idempotent $\rho$-classes:

$$
N=\operatorname{ker} \rho=\bigcup_{e \in E^{+}(S)} e \rho .
$$

We can easily prove that $a-b \in N$ for any $a, b$ in $S$, then $(a-b) c \in N$ and $c(a-b) \in N$ for any $c$ in $S$.

Definition 2.3. Let $S$ be an additive inverse semiring and $T$ a subsemiring of $S$. $T$ is said to be full, if $T$ contains all the additive idempotents of $S$.

Definition 2.4. Let $S$ be an additive inverse semiring and $T$ a subsemiring of $S$. $T$ is said to be self-conjugate, if $a \in T$ implies $-x+a+x \in T$ for all $x \in S$.

Definition 2.5. Let $S$ be an additive inverse semiring and $T$ a subsemiring of $S . T$ is said to be normal, if $T$ is full and self-conjugate. 
Proposition 2.6. Let $\rho$ be a congruence on an additive inverse semiring $S$. Then $N$ is a normal subsemiring of $S$.

Proof. Clearly $N$ is a nonempty subset of $S$. If $x \in e \rho$ and $y \in f \rho$ for some $e, f$ in $E^{+}(S)$, then $x+y \in(e+f) \rho \subseteq N, x y \in(e f) \rho \subseteq N$, and hence $N$ is a subsemiring of $S$. Certainly $N$ is full, in the sense that it contains all the idempotents of $S$. Also $N$ is self-conjugate, for $a \in e \rho$ implies that $-x+a+x \in(-x+e+x) \rho \subseteq N$. So $N$ is a normal subsemiring of $S$.

Proposition 2.7. Let $\rho$ be a congruence on an additive inverse semiring $S$. Then $\operatorname{tr} \rho$ is a normal congruence on $E^{+}(S)$.

Proof. If $(e, f) \in \operatorname{tr} \rho$, then $-a+e+a,-a+f+a, e c, f c, c e, c f$ are in $E^{+}(S)$, and hence $(-a+e+a,-a+f+a) \in \operatorname{tr} \rho$ for all $a$ in $S$ and $(e c, f c) \in \operatorname{tr} \rho,(c e, c f) \in \operatorname{tr} \rho$ for all $c$ in $S$. So $\operatorname{tr} \rho$ is a normal congruence on $E^{+}(S)$.

We might hope that the properties of a congruence $\rho$ could be completely described in terms of its kernel ker $\rho$ and its trace $\operatorname{tr} \rho$. This turns out to be the case. But before we can state a theorem we observe two connections between the kernel and trace. First, for all $a$ in $S$ and $e$ in $E^{+}(S)$, we have the following implication:

$$
a+e \in \operatorname{ker} \rho,(e,-a+a) \in \operatorname{tr} \rho \Rightarrow a \in \operatorname{ker} \rho \text {. }
$$

In fact, suppose $a+e \rho f$, where $f \in E^{+}(S)$. Then

$$
a=a+(-a)+a \rho a+e \rho f,
$$

and so $a \in \operatorname{ker} \rho$.

Also, the following implication can be obtained:

$$
a \in \operatorname{ker} \rho \Rightarrow(a+(-a),(-a)+a) \in \operatorname{tr} \rho .
$$

For suppose that $a \in e \rho$, where $e \in E^{+}(S)$. Then, as already observed, $-a \in$ $e \rho$, and it then follows that $a+(-a),(-a)+a \in e \rho$. Thus $(a+(-a),(-a)+a) \in$ $\operatorname{tr} \rho$.

We now produce an abstract version of the kernel and trace. As usual, $S$ is an additive inverse semiring.

Definition 2.8. Let $N$ be a normal subsemiring of $S$ and $\tau$ a normal congruence on $E^{+}(S)$. The pair $(N, \tau)$ is said to be a congruence pair of $S$ if

(c1) $a+e \in N$ and $(e,-a+a) \in \tau \Rightarrow a \in N$;

(c2) $a \in N \Rightarrow(a+(-a),(-a)+a) \in \tau$

for all $a \in N, e \in E^{+}(S)$.

Remark 2.9. Let $S$ be an additive inverse semiring. Then $\left(E^{+}(S), 1_{E^{+}(S)}\right)$ is a congruence pair of $S$, where $1_{E^{+}(S)}$ is an identity relation on $E^{+}(S)$. 
Before we state our main theorem, it pays to prove the following technical lemma:

Lemma 2.10. Let $S$ be an additive inverse semiring, $(N, \tau)$ a congruence pair and $a, b \in S, e \in E^{+}(S)$.

(i) If $a+e+b \in N$ and $e \tau-a+a$, then $a+b \in N$.

(ii) If $(-a+a,-b+b) \in \tau$ and $a+(-b) \in N$, then $(-a+e+a,-b+e+b) \in \tau$ for every $e$ in $E^{+}(S)$.

Proof. (i) Suppose that $a+e+b \in N$ and that $e \tau-a+a$. Then

$$
a+e+b=a+e+b+(-b)+b=a+b+(-b)+e+b=a+b+f,
$$

where $f=(-b)+e+b \in E^{+}(S)$, and so $a+b+f \in N$. Now for $(-b)+(-a)=$ $-(a+b)$, from $e \tau-a+a$ we deduce that $f \tau(-b)+(-a)+a+b=-(a+b)+a+b$, since $\tau$ is normal. Hence by (c1) it follows that $a+b \in N$.

(ii) Suppose that $(-a+a,-b+b) \in \tau, a+(-b) \in N$ and $e \in E^{+}(S)$. Then clearly (modulo $\tau$ ) $-a+e+a=-b+e+b$.

Thus the lemma is proved.

We now have our main result:

Theorem 2.11. Let $S$ be an additive inverse semiring, $\rho$ a congruence on $S$, then (ker $\rho, \operatorname{tr} \rho)$ is a congruence pair of $S$. Conversely, if $(N, \tau)$ is a congruence pair of $S$, then the relation

$$
\rho_{(N, \tau)}=\{(a, b) \in S \times S:(-a+a,-b+b) \in \tau, a+(-b) \in N\}
$$

is a congruence on $S$. Moreover, $\operatorname{ker} \rho_{(N, \tau)}=N, \operatorname{tr} \rho_{(N, \tau)}=\tau$, and $\rho_{(\operatorname{ker} \rho, \operatorname{tr} \rho)}=$ $\rho$.

Proof. By virtue of (1),(2) and proposition 2.6 and 2.7, we have in fact already established the first statement in the theorem. So suppose now that $(N, \tau)$ is a congruence pair, and let $\rho=\rho_{(N, \tau)}$ be as defined. First, we shall show that $\rho=\rho_{(N, \tau)}$ is a congruence on $S$. For this we must prove that $\rho$ is an equivalence relation. $\rho$ is a reflexive relation since $N$ is full, and is a symmetric relation since $\tau$ is symmetric and $N$ is an additive inverse subsemiring. To show that $\rho$ is transitive, let $(a, b),(b, c) \in \rho$. Then $(-a+a,-b+b),(-b+b,-c+c) \in \tau$, and so $(-a+a,-c+c) \in \tau$. Also $a+(-b), b+(-c) \in N$, from which we deduce that $N$ contains $a+(-b)+b+(-c)=a+((-b)+b)+(-c)$, where $e=(-b)+b$. since $e \tau-a+a$, we deduce from lemma 2.10(i) that $a+(-c) \in N$. Hence $(a, c) \in \rho$, and we have established the $\rho$ is an equivalence relation.

For we must prove that $\rho$ is a congruence, suppose that $a \rho b$ and let $c \in S$. Then $-a+a \tau-b+b$ and so, modulo $\tau$, $-(a+c)+(a+c)=(-c)+(-a)+a+c \equiv(-c)+(-b)+b+c=-(b+c)+(b+c)$. 
Also,

$$
\begin{aligned}
& (a+c)-(b+c)=a+c+(-c)+(-b) \\
= & a+c+(-c)+(-b)+b+(-b)=a+(-b)+(b+c+(-c)+(-b)) \in N .
\end{aligned}
$$

Thus $a+c \rho b+c$. Also, again modulo $\tau$,

$-(c+a)+(c+a)=(-a)+(-c)+c+a \equiv(-b)+(-c)+c+b=-(c+b)+(c+b)$.

by lemma 2.10(ii), and $(c+a)-(c+b)=c+a+(-b)+(-c) \in N$, since $N$ is normal. Hence $c+a \rho c+a$. For $(-x) c=-x c$ and $c(-x)=-c x$ for any $x$ in $S$, modulo $\tau$,

$$
-a c+a c=(-a+a) c \equiv(-b+b) c=-b c+b c .
$$

Also, $a c-b c=(a-b) c \in N$. Thus ac $\rho b c$. Also, again modulo $\tau$,

$$
-c a+c a=c(-a+a) \equiv c(-b+b)=-c b+c b .
$$

and $c a-c b=c(a-b) \in N$. Hence $c a \rho c b$.

We have shown that $\rho=\rho_{(N, \tau)}$ is a congruence on $S$. It is clear that if $a \in e \rho$ with $e$ in $E^{+}(S)$. then $-a+a \tau e$ and $a+e \in N$. By (c1) it follows that $a \in N$. Thus ker $\rho \subseteq N$. Conversely, if $a \in N$ then $a+e \in N$ with $e=-a+a$, and certainly $-a+a \tau-e+e$; hence $a \in e \rho \subseteq \operatorname{ker} \rho$. Hence $\operatorname{ker} \rho_{(N, \tau)}=N$.

Next, to show that $\operatorname{tr}_{(N, \tau)}=\tau$. let $e, f \in E^{+}(S)$ and suppose that $(e, f) \in$ $\rho=\rho_{(N, \tau)}$. Then

$$
e=-e+e \tau-f+f=f .
$$

Hence $\operatorname{tr} \rho \subseteq \tau$. Conversely, if $e \tau f$, then $-e+e=e \tau f=-f+f$ and $e-f=$ $e+f \in E^{+}(S) \subseteq N$; hence $(e, f) \in \rho \cap\left(E^{+}(S) \times E^{+}(S)=\operatorname{tr} \rho\right.$. That is $\operatorname{tr} \rho_{(N, \tau)}=\tau$.

To establish the final statement in the theorem, suppose first that $(a, b) \in \rho$. Then $(-a,-b) \in \rho$, and so $(-a+a,-b+b) \in \rho$. Since $-a+a,-b+b$ are idempotents, we in fact have $(-a+a,-b+b) \in \operatorname{tr} \rho$. We have shown that $\rho \subseteq \rho_{(\operatorname{ker} \rho, \operatorname{tr} \rho)}$. Conversely, suppose that $(a, b) \in \rho_{(\operatorname{ker} \rho, \operatorname{tr} \rho)}$, so that $(-a+$ $a,-b+b) \in \operatorname{tr} \rho$ and $a+(-b) \in \operatorname{ker} \rho$. Then $(a+(-b)) \rho$ is an idempotent in $S / \rho$, and so

$$
(a+(-b)) \rho=(-(a+(-b)) \rho)+((a+(-b)) \rho)=(b+(-a)+a+(-b)) \rho .
$$

Then, modulo $\rho$,

$a=a+(-a)+a \equiv a+(-b)+b \equiv b+(-a)+a+(-b)+b \equiv b+(-b)+b+(-b)+b=b$.

That is, $(a, b) \in \rho$, and this, together with the inclusion proved in the previous paragraph, gives us $\rho=\rho_{(\operatorname{ker} \rho, \operatorname{tr} \rho)}$, as required. 


\section{References}

[1] J. M. Howie, Fundamentals of semigroup theory, Oxford: Clarendon Press, 1995.

[2] J. S. Golan, The theory of semirings and their applications, Kluwer Academic Publishers, 1999.

[3] J. Zeleznikow, Regular semirings, Semigroup Forum, Vol. 23(1981), 119136.

[4] M.K. Sen and S.K. Maity, A note on orthodox additive inverse semirings, Acta Univ. Palacki. Olomuc., Fac. rer. nat., Mathematical 43 (2004), 147152.

[5] P.H. Karvellas, Inverse semirings, J. Austral Math. Soc. 18(1974), 277288.

[6] T. K. Mukherjee, M. K. Sen and S. Ghosh, On Additively Idempotent Semirings Satisfying a+ab=a, Springer, 1998, 224-233.

[7] Y.L. Zhou, The congruence-pair of orthorings, Acta scientiarum naturalium universitatis, Sunyayseni, Vol.44 No.6, 810.

[8] Y.L. Zhou, Y.Q. Guo and Z.P. Wang, The semigroup structure of orthoring, to be printed on Acta Mathematica Scientia.

[9] Y. Q. Guo, K. P. Shum, and M. K. Sen , The semigroup structure of left Clifford semiring, Acta Math. Sin. Eng. Ser. 19(2)(2003), 1-11.

Received: February, 2009 